\title{
THERMOCHEMICAL CALCULATIONS USING SERVICE- ORIENTED ARCHITECTURE IN THE WEB SERVICE FORM
}

\author{
PAVEl HorovČÁK, Ján TERPÁK* \\ Institute of Control and Informatization of Production Processes, Technical University of Košice, \\ Boženy Němcovej 3, 04200 Košice, Slovak Republic \\ * corresponding author: jan.terpak@tuke.sk
}

\begin{abstract}
The subject of this article is the service-oriented architecture utilization in the design and implementation of a web service that is intended to perform selected thermochemical calculations for chemical reactions. Computing functions allow the chemical reaction calculations, such as molar heat capacity, enthalpy, entropy and Gibbs free energy. In the next part, there is a description of each function, the method of service calling in the client application and the structure specification of outputs and error states of the service. In addition to computing functions, the web service also has a group of three information functions that characterize the purpose of the web service and its parameters, provide in tabular form a list of all web service functions and a list of all error states of the web service. The final section describes the presentation web service application with a demonstration of the specific calculations, the possibilities of using the service, and a further solution treatment.
\end{abstract}

KEYWORDS: thermochemical calculation; chemical reaction; service-oriented architecture; web service.

\section{INTRODUCTION}

The basic tasks of chemical and process engineering mainly include an analysis of existing processes, design of new processes, construction of technological equipment, optimizing the use of material and energy flows, monitoring, indirect measurement of process quantities and process control itself [1-5]. Thermochemical calculations of chemical reactions provide a basis for the quantification of processes in the chemical industry, processing of raw materials, energy and so on. This is particularly the calculation of the molar heat capacity, heat of reaction, entropy, determining the direction of a chemical reaction based on the calculation of Gibbs free energy, determination of the equilibrium constant, etc. [6, 7].

For the implementation of thermochemical calculations, there is currently a large number of software tools created using different information technologies. These technologies and program tools based on them begin at simple spreadsheets and desktop applications, continue with network applications using HTML and various server scripts and end at a service-oriented architecture [8] (SOA) through the web services [9] or enterprise services bus (ESB) [10], which is optionally also applied in the form of a cloud computing [11] (CC). The concept of cloud computing is currently one of the most popular marketing witticisms in the IT industry [11. The term Cloud with all its derivatives has been adopted as a metaphor for the Internet-based services. In work [12] several definitions and characteristics of the SOA have been given. The starting point is the basic definition, according to which the current SOA is an architecture that supports service orientation when using a web service [13. Other definitions [14, 15], specifications and characteristics [16]

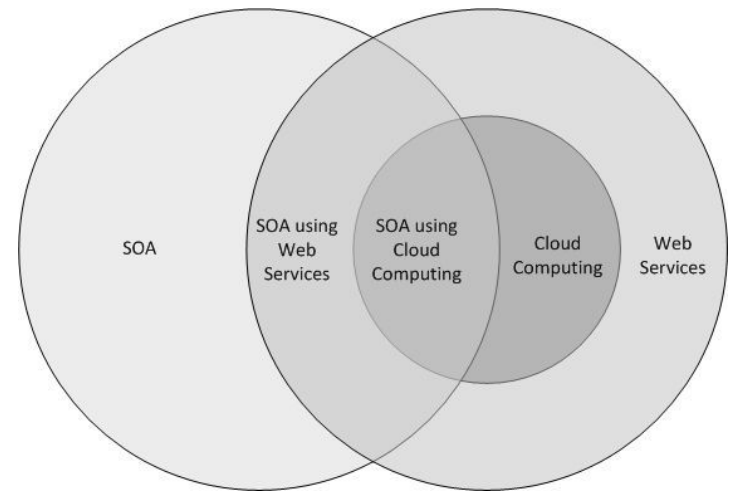

FIgURE 1. Web services and cloud computing 8

in more detail indicate the requirements, characteristics, forms, targets, platforms, interaction and applications of the SOA. The relationship between the WS, SOA, and CC can be shown in the form of the Venn diagram [8] (Fig. 1). In this diagram, the WS is concluding (encapsulating) the $\mathrm{CC}$ because the $\mathrm{CC}$ uses the WS for the purposes of connection. However, the WS are and can be used also outside CC. Such WS use can be a part of the SOA, or may not, because the SOA does not need to use only the WS for the connection purposes.

A view of the complexity of solved problems and the participation of numerous teams whose members often operate at different workplaces can be formulated by following requirements for functionality of software tools: unified management of the thermochemical data and calculations, the network user access to unified data and calculations, independence on hardware and software platforms on the user's end and the modularity of calculations in terms of their use and dissemination. 
The article is a contribution in the field of thermochemical calculations using web services and provides the possibility of its use in different areas.

\section{OvervieW OF THE CURRENT STATE}

The basis of thermochemical calculations of a chemical reaction are mainly the thermochemical data of the pure substances present in a chemical reaction. The source of thermochemical data are primarily book publications such as 17, 18, wherein the thermochemical data are shown in the form of table values, depending on the temperature.

In the case of automated calculation, the form of functions is more appropriate than table values. From publications, various forms of the functions are known [18, 19] and due to the number of substances and form of the functional dependence, it appears to be the best source known as NASA polynomials [19.

Other sources of thermochemical data are various electronic sources [20] which represent applications from a simple desktop to a network 21] application. The outcome of these applications is usually a table, which can be saved to a text file.

Analysis of the current situation also shows the number of software tools they support and implement thermochemical calculations. The best known software resources include especially FactSage [22], GWB [23], HSC Chemistry [24], MELTS [25], MTDATA [26], METSIM [27] and so on. We can also include web portals, such as CTserver [28], WebQC [29], or TEST [30, in the list of the program tools. Most software tools are commercial and some are also available for free, but with a considerably reduced substances database.

One of the few providers of the WS in the field of thermodynamic calculations is CTserver Web Services 28, which provides a total of four different services through the WSDL (Web Services Description Language) files. Services allow to obtain thermodynamic properties of minerals and their reactions using an internally consistent database of Berman [31, to calculate the thermodynamic properties of other phases and solutions in the phase library of CTserver, perform calculations of the solubility model based on Papale [32] and carry out calculations of the FeTioxygen system by Ghiorso and Evans [33. To make the data relating to the generation of free energies of reactions and minerals available, it was decided, at Geoscience Australia [34, to also create a web service that is not further specified (in the form of the WSDL). In the work 35, the authors write about the group of chemically oriented (chemoinformatics) web services (also without the WSDL) formed at Indiana University. The subject of the WS database infrastructure issues for thermochemical data [19, 36] and its application for the distributed computation of chemical equilibrium is being investigated at San Diego University in works [37, 38. The data access over the web for different calculations (chemistry, biology, etc.) in a documented WS is the subject of work 39. CDK (Chemistry Development Kit) 40 can be called a clear forerunner of web access solutions through the WS to chemical data, which addresses the client side using Java applets and in addition to the web access also allows mutual communication of the server part with the $\mathrm{R}$ system. The WS for calculations of molecular similarities using the CDK are specified in paper [41] (with broken links to the WS specification).

The use of SOA through WS, for example, calculating the reactive effectiveness of atom-diatom systems [42], is very rare. Web services herein are used for the allocation of computing capacity. Quantum reactive scattering studies of atom-diatom systems have nowadays become routine computing applications when you need to either confirm the associated potential surface energy or to accurately estimate the effectiveness of the reactive system [43].

In terms of functionality, there are software resources offering options from simple outputs to more complex tools realizing thermochemical calculations. The single outputs include, in particular, molar mass calculations of the various substances, view of the chemical formula, balance of chemical reaction providing primary thermochemical data, and the like.

More complex instruments are provided according to the system parameters (open, closed, chemical reaction, etc.), generation of state quantities (temperature, enthalpy, entropy, and the like), calculation of thermochemical properties of substances at the given temperature and pressure. They enable the acquisition of number and chemical formulas of components in the current phase or mixture, and so on.

In terms of technologies used, the individual software tools are developed using HTML, PHP, Java, SOA in WS form, and the like. Existing resources can also be divided from the point of view of an application implementation, i.e., whether it is a desktop or network application.

Within the analysis of the existing software resources, the form of communication with the user also needs to be evaluated. In the case of simple closed systems, it is mainly of visual form consisting of values and graphs. Open systems offer the possibilities from data fields to various file formats. The XML structure can be considered as most appropriate output format mainly in terms of the creation of modular software tools and their linkage to source data.

Based on analysis of existing software resources and in view of the requirements for program tools providing thermochemical data and calculations, the most suitable form seems to be the use of a service-oriented architecture through WS. Web services, among other web resources to provide data and services such as calculations, stand out mainly due to their compatibility, allowing users to use and combine different WSs, with the benefit that it does not matter in which programming resources they were created and not even on what software platform the service is implemented. 


\section{THERMOCHEMICAL CALCULATIONS}

Selected thermochemical calculations of chemical reaction realised in the form of a web service and described in this article are based on the following general chemical reaction in the form

$$
0=\sum_{j=1}^{m} \nu_{j} N_{j}
$$

where $\nu_{j}$ is the stoichiometric coefficient of substance $N_{j}$ and $m$ is the number of substances in the reaction. The value of $\nu_{j}$ is positive in the case of a product and is negative in case of a reactant.

The molar heat capacity of the general chemical reaction is given by

$$
\Delta C_{p}^{\circ}(T)=R \sum_{i=1}^{7} \Delta a_{i} T^{i-3},
$$

where $R$ is the universal gas constant (8.314 $\left.\mathrm{J} \mathrm{K}^{-1} \mathrm{~mol}^{-1}\right), \Delta a_{i}$ is the coefficient for a given chemical reaction based on the coefficients of the NASA polynomial for pure substances [12, 19] and $T$ is the temperature $(\mathrm{K})$. The reference state of the substances is generally taken to be the thermodynamically stable state at a temperature $T_{0}=298.15 \mathrm{~K}$.

Coefficient $\Delta a_{i}$ is calculated as follows

$$
\Delta a_{i}=\sum_{j=1}^{m} \nu_{j} a_{i, j}
$$

where $a_{i, j}$ is $i$-th coefficient of the NASA polynomial for $j$-th substance of the chemical reaction (1).

Calculation of the reaction enthalpy for a given chemical reaction is based on the integral of the molar heat capacity

$$
\Delta_{r} H^{\circ}(T)=\Delta_{r} H^{\circ}\left(T_{0}\right)+\int_{T_{0}}^{T} \Delta C_{p}^{\circ}(T) d T,
$$

where $\Delta_{r} H^{\circ}\left(T_{0}\right)$ is reaction the enthalpy for a given chemical reaction at a temperature $T_{0}$ and is calculated according to the formula

$$
\Delta_{r} H^{\circ}\left(T_{0}\right)=\sum_{j=1}^{m} \nu_{j} H_{j}^{\circ}\left(T_{0}\right),
$$

where $H_{j}^{\circ}\left(T_{0}\right)$ is the enthalpy of the pure substance $j$-th at a temperature $T_{0}$.

The calculation of the entropy change for a given chemical reaction is based on the integral of the proportion of changes in molar heat capacity and temperature

$$
\Delta_{r} S^{\circ}(T)=\Delta_{r} S^{\circ}\left(T_{0}\right)+\int_{T_{0}}^{T} \frac{\Delta C_{p}^{\circ}(T)}{T} d T,
$$

where $\Delta_{r} S^{\circ}\left(T_{0}\right)$ is the entropy change for a given chemical reaction at a temperature $T_{0}$ and is calculated according to the formula

$$
\Delta_{r} S^{\circ}\left(T_{0}\right)=\sum_{j=1}^{m} \nu_{j} S_{j}^{\circ}\left(T_{0}\right)
$$

where $S_{j}^{\circ}\left(T_{0}\right)$ is the entropy of the pure substance $j$-th at a temperature $T_{0}$.

Gibbs free energy is calculated on the basis of changes in the enthalpy (4) and entropy (6) using the equation

$$
\Delta_{r} G^{\circ}(T)=\Delta_{r} H^{\circ}(T)-T \Delta_{r} S^{\circ}(T) .
$$

Based on the equation

$$
\Delta_{r} G^{\circ}(T)=-R T \ln K
$$

the value of the logarithm of the equilibrium constant is equal to

$$
\ln K=-\frac{\Delta_{r} G^{\circ}(T)}{R T}
$$

Based on the equation (4) for calculating the enthalpy and van't Hoff reaction isobar

$$
\left(\frac{\partial \ln K}{\partial T}\right)_{p}=\frac{\Delta_{r} H^{\circ}(T)}{R T^{2}},
$$

and after integration, we obtain the relation for the equilibrium constant logarithm

$$
\begin{gathered}
\ln K(T)=\ln K\left(T_{0}\right)-\frac{\Delta_{r} H^{\circ}(0)}{R}\left(\frac{1}{T}-\frac{1}{T_{0}}\right) \\
+\frac{\Delta a_{1}}{2}\left(\frac{1}{T^{2}}-\frac{1}{T_{0}^{2}}\right)+\Delta a_{2}\left(\frac{1}{T_{0}}-\frac{1+\ln \frac{T}{T_{0}}}{T}\right) \\
+\Delta a_{3} \ln \frac{T}{T_{0}}+\sum_{i=4}^{7} \Delta a_{i} \frac{\left(T^{i-3}-T_{0}^{i-3}\right)}{(i-2)(i-3)}
\end{gathered}
$$

where

$$
\begin{aligned}
\Delta_{r} H^{\circ}(0)=\Delta_{r} & H^{\circ}\left(T_{0}\right) \\
& -R\left(-\frac{\Delta a_{1}}{T_{0}}+\sum_{i=3}^{7} \frac{\Delta a_{i} T_{0}^{i-2}}{i-2}\right) .
\end{aligned}
$$

The equilibrium constant for the general form of a chemical reaction (1) can be expressed using activities [7]. If we set the activities of condensed components of the chemical reaction equal to one and the gaseous components are expressed by means of partial pressures, the equilibrium constant takes the form

$$
K=\prod_{j=1}^{m}\left(\frac{p_{j}^{\circ}}{p^{\circ}}\right)^{\nu_{j}}
$$

where $p^{\circ}$ is the normal pressure $(101325 \mathrm{~Pa}), p_{j}^{\circ}$ are partial pressures.

Based on the known value of the equilibrium constants and the specified total pressure, we can calculate the equilibrium partial pressure, or equilibrium composition, of the gas component. From the point of view of the number of gas components on the side of products, or reactants, and of the number of moles, different specific cases can occur. In the following, we consider only the cases where in the chemical reaction we have: 


\begin{tabular}{cccccc}
\hline$\nu_{i}$ & $\nu_{j}$ & $c_{3}$ & $c_{2}$ & $c_{1}$ & $c_{0}$ \\
\hline$n$ & $n$ & 0 & 0 & $1+\sqrt[n]{K}$ & $-\sqrt[n]{K} p_{c}$ \\
\hline$n$ & $2 n$ & 0 & 1 & $\sqrt[n]{K} p^{\circ}$ & $-\sqrt[n]{K} p^{\circ} p_{c}$ \\
\hline$n$ & $2 n$ & 1 & 0 & $\sqrt[n]{K}\left(p^{\circ}\right)^{2}$ & $-\sqrt[n]{K}\left(p^{\circ}\right)^{2} p_{c}$ \\
\hline $2 n$ & $3 n$ & 1 & $-\sqrt[n]{K}$ & $2 \sqrt[n]{K} p^{\circ} p_{c}$ & $-\sqrt[n]{K} p^{\circ}\left(p_{c}\right)^{2}$ \\
\hline
\end{tabular}

TABLE 1. The equation coefficients 17 for the number of moles of reactant $\left(\nu_{i}\right)$ and product $\left(\nu_{j}\right)$.

- one gaseous product,

- one gaseous reactant,

- one gaseous product and one gaseous reactant.

In the first case, where one gaseous product $N_{j}$ with the number of moles of $\nu_{j}$ occurs in the chemical reaction, we can express the equilibrium constant as follows

$$
K=\left(\frac{p_{j}^{\circ}}{p^{\circ}}\right)^{\nu_{j}}, \quad \text { resp. } \quad p_{j}^{\circ}=p^{\circ} \sqrt[\nu_{j}]{K}
$$

In the second case, where one gaseous reactant $N_{i}$ with the number of moles of $\nu_{i}$ occurs in the chemical reaction, it is essentially the first case that the chemical reaction takes place in the opposite direction. The equilibrium constant has the form

$$
K=\left(\frac{p_{i}^{\circ}}{p^{\circ}}\right)^{\nu_{i}}, \quad \text { resp. } \quad p_{i}^{\circ}=\frac{p^{\circ}}{\sqrt[\nu_{i}]{K}}
$$

In the third case, where one gaseous product $N_{j}$ with the number of moles of $\nu_{j}$ and one gaseous reactant $N_{i}$ with the number of moles of $\nu_{i}$ occurs in the chemical reaction and the sum of the partial pressures is equal to the total pressure $\left(p_{c}=p_{i}^{\circ}+p_{j}^{\circ}\right)$, then it is necessary to solve the following equation

$$
c_{3}\left(p_{j}^{\circ}\right)^{3}+c_{2}\left(p_{j}^{\circ}\right)^{2}+c_{1} p_{j}^{\circ}+c_{0}=0,
$$

where coefficients $c_{i}$ are given in the Tab. 1

The result of the equation (17) is the partial pressure of the gaseous product $p_{j}^{\circ}$, or mole fraction $x_{j}=p_{j}^{\circ} / p_{c}$, or mole percent. The calculations represent a generalization of the calculations for gaseous components while generally only solutions for specific cases are found in the literature [6].

Finding direction of a chemical reaction or Gibbs free energy for known initial partial pressures is based on the following equation

$$
\begin{aligned}
\Delta_{r} G^{\circ}(T)=\Delta_{r} & G^{\circ}\left(T_{0}\right) \\
& +R T \ln \left(\left(p^{\circ}\right)^{-\Delta \nu} \prod_{j=1}^{m}\left(p_{j}^{\circ}\right)^{\nu_{j}}\right),
\end{aligned}
$$

where the calculation of $\Delta_{r} G^{\circ}(T)$ is according to (9), and the equilibrium constant is determined by van't Hoff reaction isobar 12 . The value of $\Delta \nu$ represents the change in the total quantity of moles of substances.

\section{WeB SERVICE DESIGN}

The purpose of the web service named as ThermoChemDC (Thermo Chemical Data Calculations) is to do selected thermochemical calculations of a chemical reaction. The WS provides two groups of calculations. The first group includes the calculation of the change of the molar heat capacity (2), the calculation of the heat of reaction (4), the calculation of the entropy change (6), the calculation of Gibbs free energy (8) and the calculation of the logarithm of the equilibrium constant 10 . The calculations can be made for a specified temperature or a temperature interval. The second group includes the calculation of the logarithm of the equilibrium constant (11), the calculation of the equilibrium composition (14) and the calculation of Gibbs free energy (18) using the van't Hoff reaction isobar (11). Above-mentioned calculations can be performed for a given temperature, pressure or partial pressure or their intervals. Other WS functions include the balance control of chemical reaction and the decomposition of both sides of a chemical reaction on its individual components. In addition to computing functions, the WS also has a group of three information functions that are designed as multi-lingual. These functions characterize the purpose of WS ThermoChemDC and its parameters, provide in tabular form a list of all functions of the WS and also list of all error states of WS ThermoChemDC.

In its activities, the WS uses additional web services that have been proposed and implemented in our department. WS ThermPropDC [12] provides thermochemical properties of chemical substances (1154 substances and their 1817 phases) for the purposes of individual calculations. WS ChemForm [44] is used to convert standard notation of the chemical formula stored in the ASCII form to the HTML format. Web service ErrorService is designed for the selection of error status meaning for a specified WS using its numeric identifier for the specified error's code, and in the chosen language.

WS ThermoChemDC directly uses the WS ThermPropDC based on the use of NASA resources (NASA Polynomials) [12, 19, 36] in all calculations, which include also other published dependences [6, [17, 45, 46] and are supplemented with the CAS [47] 


\begin{tabular}{|c|c|c|}
\hline$\#$ & Meaning of function & Function parameters \\
\hline 1 & Enthalpy & Reaction, Language, Temperature $[\mathrm{K}]$ \\
\hline 2 & Enthalpy for the temperature interval & $\begin{array}{l}\text { Reaction, Language, Temperature1 }[\mathrm{K}] \\
\text { Temperature } 2[\mathrm{~K}] \text {, Step }[\mathrm{K}]\end{array}$ \\
\hline 3 & Gibbs energy & Reaction, Language, Temperature $[\mathrm{K}]$ \\
\hline 4 & $\begin{array}{l}\text { Gibbs energy for the temperature } \\
\text { interval }\end{array}$ & $\begin{array}{l}\text { Reaction, Language, Temperature }[\mathrm{K}] \\
\text { Temperature } 2[\mathrm{~K}] \text {, Step }[\mathrm{K}]\end{array}$ \\
\hline 5 & Entropy & Reaction, Language, Temperature $[\mathrm{K}]$ \\
\hline 6 & Entropy for the temperature interval & $\begin{array}{l}\text { Reaction, Language, Temperature }[\mathrm{K}] \\
\text { Temperature } 2[\mathrm{~K}] \text {, Step }[\mathrm{K}]\end{array}$ \\
\hline 7 & Logarithm of the equilibrium constant & Reaction, Language, Temperature $[\mathrm{K}]$ \\
\hline 8 & $\begin{array}{l}\text { Logarithm of the equilibrium constant } \\
\text { for the temperature interval }\end{array}$ & $\begin{array}{l}\text { Reaction, Language, Temperature1 }[\mathrm{K}] \\
\text { Temperature } 2[\mathrm{~K}] \text {, Step }[\mathrm{K}]\end{array}$ \\
\hline 9 & Molar heat capacity & Reaction, Language, Temperature $[\mathrm{K}]$ \\
\hline 10 & $\begin{array}{l}\text { Molar heat capacity for the temperature } \\
\text { interval }\end{array}$ & $\begin{array}{l}\text { Reaction, Language, Temperature1 }[\mathrm{K}] \\
\text { Temperature } 2[\mathrm{~K}] \text {, Step }[\mathrm{K}]\end{array}$ \\
\hline 11 & Control of chemical reactions balance & Reaction, Language \\
\hline 12 & Decomposition of chemical reaction & Reaction, Language \\
\hline 13 & $\begin{array}{l}\text { Logarithm of the equilibrium constant } \\
\text { (van't Hoff) }\end{array}$ & Reaction, Language, Temperature $[\mathrm{K}]$ \\
\hline 14 & $\begin{array}{l}\text { Logarithm of the equilibrium constant } \\
\text { for the temperature interval (van't Hoff) }\end{array}$ & $\begin{array}{l}\text { Reaction, Language, Temperature1 }[\mathrm{K}] \\
\text { Temperature } 2[\mathrm{~K}] \text {, Step }[\mathrm{K}]\end{array}$ \\
\hline 15 & Equilibrium composition & Reaction, Language, Temperature $[\mathrm{K}]$, Pressure $[\mathrm{Pa}]$ \\
\hline 16 & $\begin{array}{l}\text { Equilibrium composition for the } \\
\text { pressures interval }\end{array}$ & $\begin{array}{l}\text { Reaction, Language, Temperature }[\mathrm{K}] \text {, Pressure1 }[\mathrm{Pa}] \text {, } \\
\text { Pressure2 }[\mathrm{Pa}] \text {, Step }[\mathrm{Pa}]\end{array}$ \\
\hline 17 & Gibbs energy (van't Hoff) & $\begin{array}{l}\text { Reaction, Language, Temperature }[\mathrm{K}] \text {, Pressure }[\mathrm{Pa}] \\
\text { Partial Pressure }[\mathrm{Pa}]\end{array}$ \\
\hline 18 & $\begin{array}{l}\text { Gibbs energy for the pressures interval } \\
\text { (van't Hoff) }\end{array}$ & $\begin{array}{l}\text { Reaction, Language, Temperature }[\mathrm{K}] \text {, Pressure }[\mathrm{Pa}] \\
\text { Partial Pressure1 }[\mathrm{Pa}], \text { Partial Pressure } 2[\mathrm{~Pa}] \text {, Step }[\mathrm{Pa}]\end{array}$ \\
\hline
\end{tabular}

TABLE 2. List of web service ThermoChemDC functions

parameter and with one or more names of substances in two languages. In some cases, the value of 000000 is used for the CAS identifier, which indicates the fact that it failed to detect CAS value for a given substance at any website. The CAS identifier is optionally stored in the database infrastructure of the WS as a link to the appropriate website 4854. Similarly, you can choose to save the formula of the substance in a standard ASCII form or in a HTML format (the form of the upper/lower index), which ensures the WS ChemForm [44]. General administration of the database infrastructure of the WS ThermPropDC provides a standalone application admin_tp. This application allows a gradual creation of all tables in the operation and preparation service's database, populating tables with NASA, CAS data and the names from the relevant files, transfer complete records to the operation database, optionally manually completing the CAS identifier and the names of substances and/or perform a backup of the database into a file.

WS ThermoChemDC is implemented in a PHP development environment (required PHP version 5 and above) and as a database system, it uses MySQL in version 5 also. The current version of the WS with a class named ThermoChemDC includes a total of 52 methods, while five of them are service interfaces and are also specified in the file ThermoChemDC.wsdl. Other methods are internal and intended for different calculations or control, creating XML structures, auxiliary, declaratory, folding tasks, a communication with the database system or are complementary.

\section{The METHODS AND FUNCTIONS OF WEB SERVICE}

The WS ThermoChemDC provides users with a total of five methods through the WSDL file. The first two 


\begin{aligned} & \hline$\#$ Meaning of error \\ & \hline 1 One of the reactants is not in the database \\ & 2 One of the products is not in the database \\ & 3 The specified temperature is outside the permitted interval (left side — reactants) \\ & 4 Syntax error in the reaction writing \\ & 5 Incorrect reaction - reactants and products do not contain the same chemical elements \\ & 6 Calling a non-existent function of the web service - method getThermoChemCalc \\ & 7 Incorrect parameters (1 or more) of web service calling - method getThermoChemCalc \\ & 8 Used web service is off-line \\ & 9 Connection error to database (db server): Access denied for user \\ & 10 Error when selecting error meaning from db table [ws_errors] \\ & 11 Incorrect reaction balance - reactants and products do not contain the same number \\ & of chemical elements \\ & 12 The reaction does not contain any gaseous compound \\ & 13 The numbers of gaseous components of the reaction greater than 1 are not yet solved \\ & 14 Calling a non-existent function of the web service - method getParcPresCalc \\ & 15 Incorrect parameters (1 or more) of web service calling - method getParcPresCalc \\ & 16 The first parameter of the cubic parabola respectively Kp1 is equal to zero \\ & 17 Error when selecting function meaning from db table [ws_functions] \\ & 18 Error when selecting function parameters from db table [ws_funcparams] \\ & 19 The specified temperature is outside the permitted interval (right side - products) \\ & \hline\end{aligned}

TABLE 3. List of web service ThermoChemDC error states

methods are computational - getThermoChemCalc and getParcPresCalc, the other three methods are informational - getHelp, getWsFunc, and getError. Each calculation method can operate in several modes and thus perform the various functions. Calculation methods have from three to six parameters. The first three parameters are required, the first parameter specifies the specific function of WS, the second parameter contains a chemical reaction and the third parameter defines the language of outputs or error messages of the WS. Other parameters are related to the desired function of the calculation method according to Tab. 2. The method getThermoChemCalc is used to solve the functions $1-12$, the method getParcPresCalc to solve the functions $13-18$.

The method getHelp provides the user of the WS with a brief description of the service - purpose, WSDL file, list of methods, their purpose and parameters, description of their outputs and corresponding XML structures, and other WS used within the service itself. The method getWsFunc provides a list of all WS functions together with parameters of these functions in a tabular form - Tab. 2 The first column is the function code; the second column is a more detailed description of the relevant service function, and a list of its parameters. The return value of getError method is a list of all error states of ThermoChemDC WS, which is also implemented in a tabular form — Tab. 3. The first column is the error code; in the second column, there is a more detailed description of the service's error state. All three informational methods have one parameter $\$$ lang that defines the selected language of the output of above methods.

\section{WEB SERVICE CALL}

WS ThermoChemDC is available to clients through a WSDL file, which is located at http://omega.tuke sk/wsdl/ThermoChemDC.wsdl. This file specifies all the attributes required for the WS call. The WS can be used either in a separate client application or in another WS. Calling the WS consists of a sequence of two steps. The first step is a instantiated object that represents the client part of the WS. To create an instance, a WSDL service file is used. The second step is the appropriate method call, which is the WS function with the corresponding parameters. The object instance created in the previous step is used for the calling. The WS calling is supported by all current development environments (whether in the client application or in other WS). These are primarily web development environments (e.g., PHP, Java, ASP, Ajax), but they also include desktop development environments (e.g., MATLAB). The above-mentioned sequence of the WS call steps is then dependent on the chosen development environment for the implementation of the WS call. For an illustration of calling the WS we describe the procedures in a development environment PHP. Step one, create an object instance, has the form:

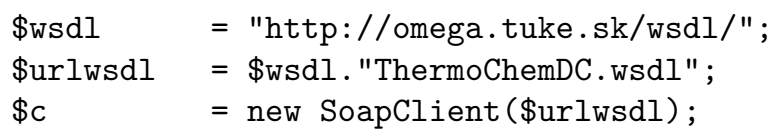

where \$urlwsdl indicates the location of the WSDL file of the WS (its URL) and \$c is an instance of an object to interact with the WS. The second step is to call the desired method and/or the WS function through the instance \$c and, after entering specific 


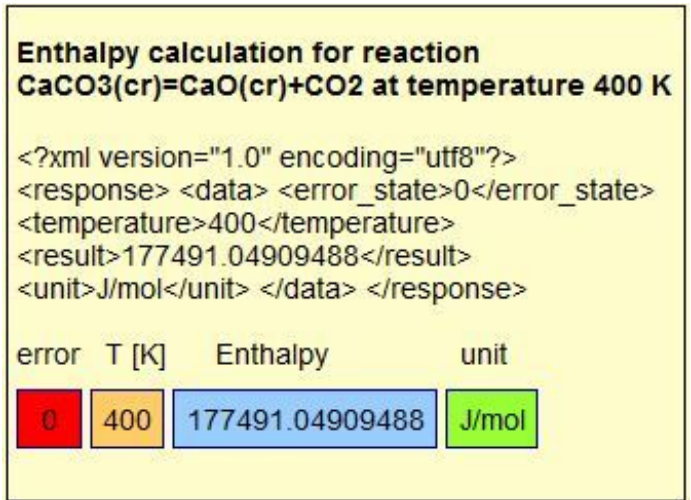

Figure 2. Sample output of a single client of ThermoChemDC WS

parameters, for example, to calculate the enthalpy $(\$ r=1)$, may take the form:

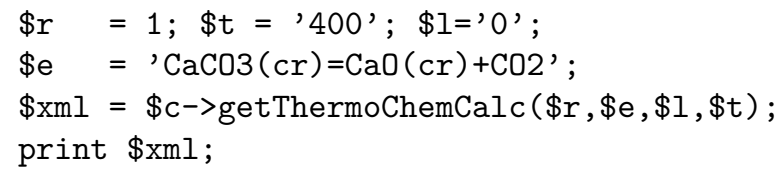

Because the WS returns as the output value the XML structure ( $\$ \mathrm{xml})$, the easiest way of its presentation is the direct visual display of this structure. Variables $\$ r, \$ e, \$ 1, \$ t$ are calling parameters of the selected WS function, where $\$ r$ is the selection of the function (WS mode), \$e contains a chemical equation, \$1 is the language and $\$ \mathrm{t}$ is the temperature.

The appearance of the presentation results on the screen justifies using a CSS file, in which to each specific element of the output XML structure of the web service, a corresponding way of view is prescribed (such as appropriate colourfulness of individual items). Those items of the XML structure that should not, for any reason, be displayed can be identified as 'display: none'. The simplicity of creating a client application illustrates a sample of an enthalpy calculation (mode 1 ) for the reaction ' $\mathrm{CaCO} 3(\mathrm{cr})=\mathrm{CaO}(\mathrm{cr})+\mathrm{CO} 2$ ' for a given temperature $(400 \mathrm{~K})$. The script has the form:

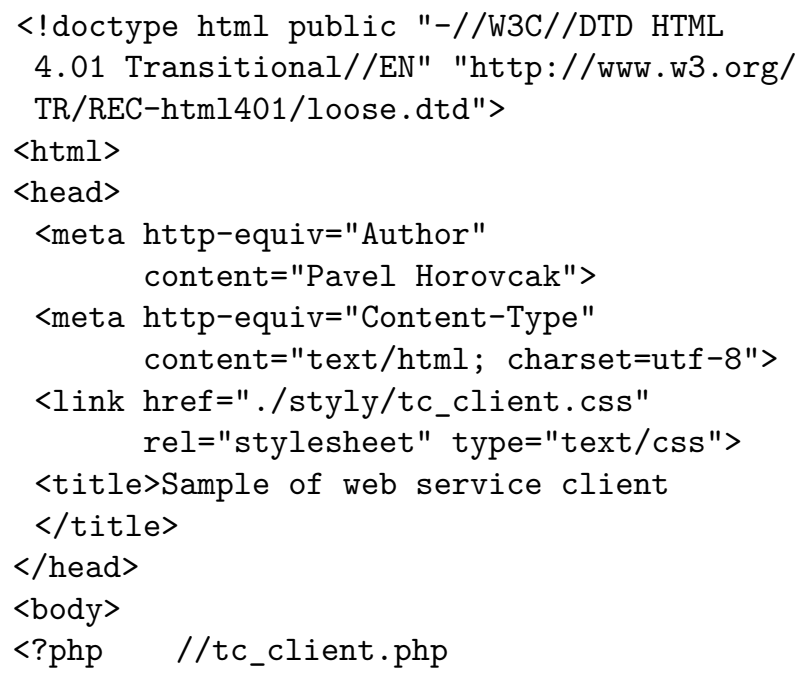

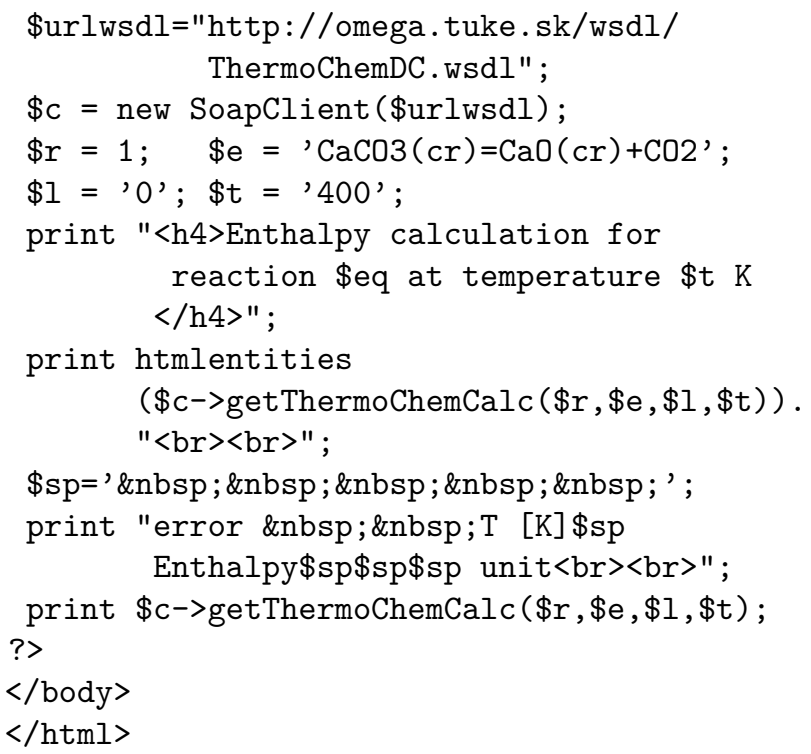

and it is also available on http://omega.tuke.sk/ tc/client_tc.php The output of this sample is shown in Fig. 2 The line of the PHP code containing the function htmlentities (...) only serves to display the XML structure (for this article), which is the WS response to the submitted request (usually does not need to be placed in the script). To visualize the result of this example, a simple CSS file is composed (http://omega.tuke.sk/tc/styly/ client_tc.css). The creation of a client in a PHP environment requires the version 5 and above. In the current distribution of Linux, this version is already included.

\section{OUtPut AND ERROR STATES OF WEB SERVICE}

The WS output (the return value of the method getThermoChemCalc() or getParcPresCalc()) is one of the six XML structures that may take the form as shown on http://omega.tuke.sk/tc/docs/ struktury_ws0.pdf Five structures are the result of one of the functions of the WS, one structure contains an error message. Successfulness of the WS processing indicates the element <error_state>, which, in case of an error, has a value 1 and in other cases acquires a value 0 . Element <error_state $>$ is a part of each output XML structure. The first structure in the illustration is the most frequent structure containing the numerical result and calculation unit of the selected WS function at the specified temperature (excluding the calculation of logarithm of the equilibrium constant, which is dimensionless, where as a unit the value " 1 " is listed). The second one is an error structure that contains the code and description of the error state in the selected language and the values of all six specified parameters of the WS call. After that follows a structure that is returned by the function to control the balance of components in the reaction (containing the individual elements of the reaction and their quantity that is the same on both 
sides of the reaction), then follows a structure that is returned by the function of the decomposition reaction (the moles number of all components on both sides of the reaction, the element $<$ condensed_phase $>$ indicates the componentâẮ́s affiliation to the condensed phase). Another structure that is the output of the function for calculating the equilibrium composition for a given temperature and pressure (the 15-th and 16 -th function). The last structure returns the function to the calculation of Gibbs free energy for a given temperature, pressure and partial pressure by van't Hoff reaction isobar (function 17-th and 18-th).

Error states are the result of the WS input parameters checking or execution of individual modes of the WS. A centralized way to control the input parameters of the WS required a suitable solution of error states handling on the service server side and their sending to the client side. The indication of an error state to the client is realized by an error XML structure. Tab. 3 illustrates a list of various error states.

\section{Presentation and utilization OF WEB SERVICE}

For the purposes of presentation of the WS functions, the client application ThermoChemDC_App (http://omega.tuke.sk/tc) is assembled, which allows to enter a chemical reaction, calculate the enthalpy (heat of reaction), heat capacity, entropy, Gibbs free energy and the logarithm of the equilibrium constant of the reaction, the execution of the chosen calculation for a given temperature or temperature interval, calculate the logarithm of the equilibrium constant of the reaction, the calculation of the equilibrium composition and calculation of Gibbs free energy by van't Hoff reaction isobar, the execution of the chosen calculation for a given temperature or partial pressure respectively its intervals, obtaining information on using the WS, obtaining information on WS ThermoChemDC, listing functions list of WS ThermoChemDC, generate a list of error states of WS ThermoChemDC, and a list of information about ThermoChemDC_App client application. Menu items "Calculation", "About Web services", "WS functions" and "WS errors" directly use the WS ThermoChemDC. Other menu items are used only within the application ThermoChemDC_App.

The ThermoChemDC_App application displays the results of calculations in a tabular form and the interval of temperatures or pressures in a form of a graph, which is produced with the use of an object-oriented PHP library JpGraph [55]. ThermoChemDC_App application is compiled in an environment HTML5 using cascading style sheets CSS3 as a multilingual option (specifically, there are three languages - EN, SK, CZ). The ThermoChemDC_App client application displays the formula in the correct (HTML) format, using the WS ChemForm 44.

Using a ThermoChemDC WS, or ThermoChemDC_App application, can be illustrated in the

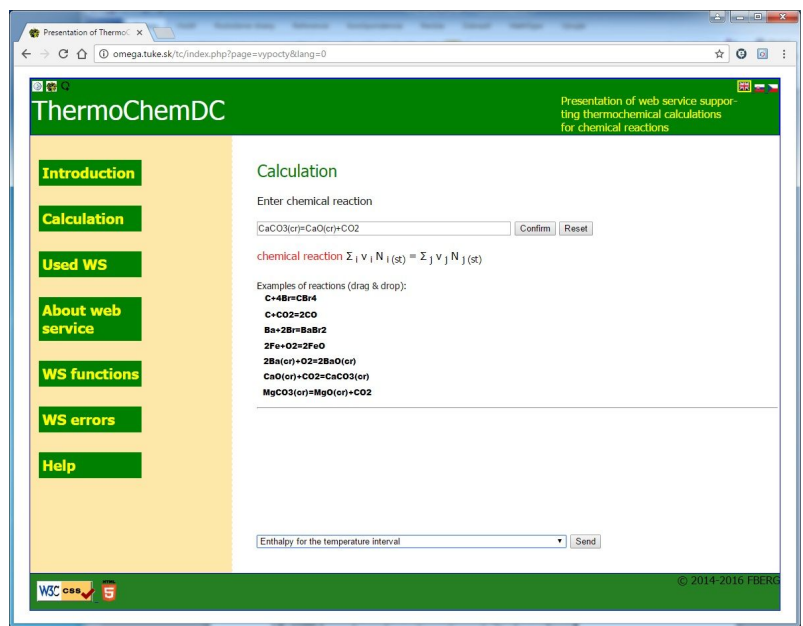

Figure 3. Screenshot of the ThermoChemDC_App application

carbonate decomposition, which is a relatively common occurrence in the different technologies covering the area of the raw materials processing, such as e.g., sintering ore, coke, blast furnace process, the processing of limestone, and the like.

Therefore, based on a chemical reaction

$$
\mathrm{CaCO}_{3(c r)} \rightarrow \mathrm{CaO}_{(c r)}+\mathrm{CO}_{2}
$$

we implement thermochemical calculations - the enthalpy for temperature from 400 to $1200 \mathrm{~K}$ with increments of $100 \mathrm{~K}$, or an equilibrium composition for a pressure from 101325 to $1013250 \mathrm{~Pa}$ with increments of $10000 \mathrm{~Pa}$ for temperatures $1000 \mathrm{~K}$ and $1100 \mathrm{~K}$.

After running the web application ThermoChemDC_App and selecting "Calculation", it is necessary to enter a chemical reaction in the form 119 and perform the send option by pressing "Confirm". Subsequently, the calculation will select, for example, "Enthalpy for the temperature interval" and the selection is confirmed by pressing the "Send" button (Fig. 3).

Subsequently, the boundaries of the interval of temperatures with an increment of the interval are filled in and sent after pressing the "Send" button, then the table and graph are shown (Fig. 4). Also other thermochemical calculations are similarly realized.

In the case of the limestone decomposition 19 it is an endothermic reaction and the gaseous substance $\left(\mathrm{CO}_{2}\right)$ is only on the product side. Therefore, the equilibrium composition of $\mathrm{CO}_{2}$ with increasing pressure decreases. In contrast, the value of the $\mathrm{CO}_{2}$ equilibrium composition at $1000 \mathrm{~K}$ is lower (Fig. 5 ) than the one at the temperature of $1100 \mathrm{~K}$ (Fig. 6) at the same pressure.

The assembled WS provides a wide range of applications under those sub-functions. The web service can be used directly as a part of the client application in a variety of network and desktop environments (e.g., PHP, Ajax, ASP, MATLAB, Java, Perl, Octave), but can also be used as a part of another WS. For 


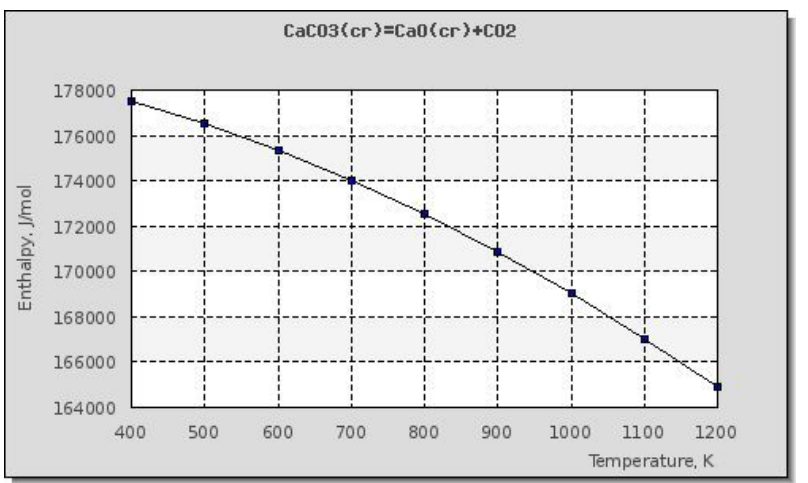

Figure 4. Enthalpy as a function of the temperature

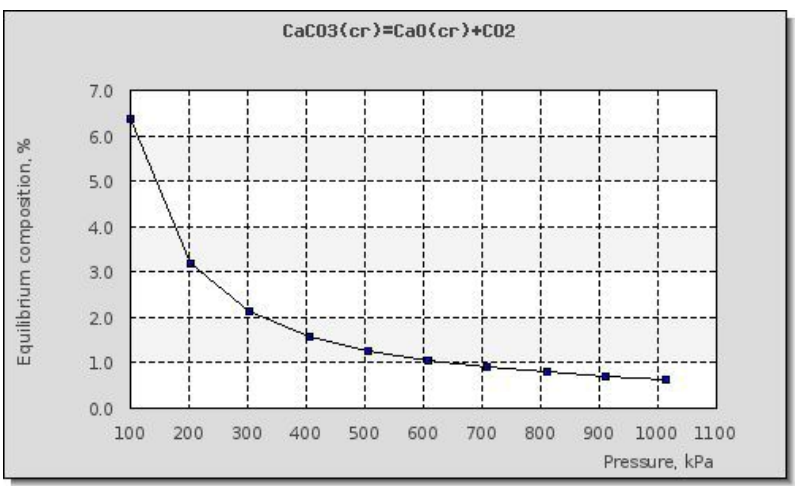

FiguRE 5. The equilibrium composition of the $\mathrm{CO}_{2}$ depending on pressure at $1000 \mathrm{~K}$

illustration, we mention some of the possible fields of application - process modelling in which heat is present, cooling off, phase changes, chemical reactions and other calculations in which the thermochemical properties of the substances are necessary, and the thermochemical calculations for chemical reactions. Furthermore, WS provides easy access to data and supports distributed computing. In the case of updating data in NASA polynomials, through the application admin_tp is realized an immediate update of service's database infrastructure. The WS can also be used as an effective tool for teaching students in the educational process as well as in their individual work.

\section{Conclusion}

The paper presents a design of the web service ThermoChemDC for the implementation of selected thermochemical calculations for chemical reactions. These calculations include the calculation of changes in the molar heat capacity, heat of reaction, calculation of entropy change, Gibbs free energy change, and calculation of the logarithm of the equilibrium constant for a given temperature or the temperatures interval. Next, there are calculation of the logarithm of the equilibrium constant, calculation equilibrium composition and the calculation of Gibbs free energy by van't Hoff reaction isobar, which may be implemented for a given temperature, pressure or the partial pressure, or their

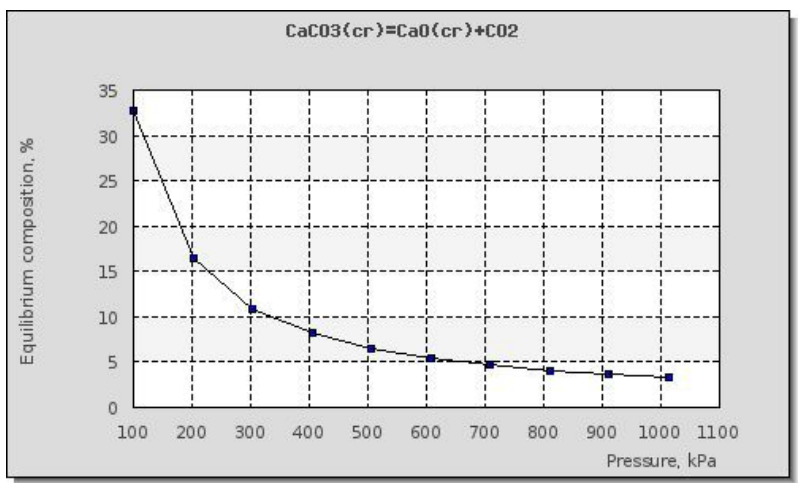

Figure 6. The equilibrium composition of the $\mathrm{CO}_{2}$ depending on pressure at $1100 \mathrm{~K}$

intervals. The mentioned calculations are carried out by particular functions of the web service. The output of the web service is XML structures corresponding to these functions, or an error structure that may arise either due to incorrect input parameters of the web service call or during the calculation itself. For purposes of presentation of the web service, a multilingual stand-alone application was created that includes all of the above calculations, more detailed description of the web service, the list of functions and error states of the web service and characteristics of other web services used. The output of this application is a result in a tabular form and, in the case of interval of temperatures or pressures, also in a graphical form. The database infrastructure of the service contains a total of 1154 substances and their 1817 phases and using the admin application admin_tp at any time, it is possible to update the database. A benefit of the service is the support of distributed computing in a variety of customer environments, interoperability support within the service-oriented architecture, the possibilities for the composition service in developing other services and simulation models and applications within the cloud. In a future solution, we propose the separation of list of functions and its connection (and administration) by a single web service. We also consider the independence of some partial supporting calculations and their realization in the form of a web service and thus making them available to other services, simulation models or calculations. Similarly, in a future solution, we also plan to add some other calculation options (more reactions at the same time) into the presentation of the web service application.

\section{ACKNOWLEDGEMENTS}

This work was partially supported by the Slovak Research and Development Agency under the contract No. APVV0482-11, APVV-14-0892, APVV SK-PL-2015-0038 and by project VEGA 1/0273/17.

\section{REFERENCES}

[1] M. Žecová, J. Terpák. Heat conduction modeling by using fractional-order derivatives. Applied Mathematics 
and Computation 257:365-373, 2015. DOI:10.1016/j.amc.2014.12.136

[2] J. Kačur, M. Durdán, M. Laciak, P. Flegner. Impact analysis of the oxidant in the process of underground coal gasification. Measurement 51(1):147-155, 2014. DOI:10.1016/j.measurement.2014.01.036

[3] K. Kostúr, M. Laciak, M. Durdán, et al. Low-calorific gasification of underground coal with a higher humidity. Measurement 63:69-80, 2015. DOI:10.1016/j.measurement.2014.12.016

[4] J. Terpák, L. Dorčák, V. Maduda. Combustion process modelling and control. Acta Montanistica Slovaca 12(3):238-242, 2007. http:

//actamont.tuke.sk/pdf/2007/n3/11terpak.pdf.

[5] M. Laciak, P. Flegner, P. Horovčák, et al. System of indirect measurement temperature of melt with adaptation module. In Proceedings of the 16th International Carpathian Control Conference, pp. 277-281. IEEE, 2015. DOI:10.1109/CarpathianCC.2015.7145088

[6] P. E. Liley. 2000 Solved Problems in Mechanical Engineering Thermodynamics. McGraw-Hill, 1989. ISBN 0-07-037863-0.

[7] E. B. Smith. Chemical Thermodynamics. Imperial College Press, 2014. ISBN 978-1-78326-336-3.

[8] D. K. Barry. Web Services, Service-Oriented Architectures, and Cloud Computing. 2000. http://www.service-architecture.com/articles/ cloud-computing/web_services_and_cloud_ computing.html

[9] W3C working group: Web Services Architecture. 2004. http://www .w3.org/TR/ws-arch/

[10] D. A. Chappell. Enterprise Service Bus. 2006. http://archive.visualstudiomagazine.com/books/ chapters/0596006756.pdf.

[11] P. Mell, T. Grance. The NIST Definition of Cloud Computing. Recommendations of the National Institute of Standards and Technology Special Publication 800-145, 2011. http://faculty.winthrop.edu/ domanm/csci411/Handouts/NIST.pdf

[12] P. Horovčák, J. Terpák. Termochemické vlastnosti substancií formou webovej služby [Thermochemical properties of substances on web]. Chemické listy 107(2):136-145, 2013. http://www. chemicke-listy . cz/docs/full/2013_02_136-145.pdf

[13] T. Erl. SOA: Principles of Service Design. Prentice Hall, 2007. ISBN 978-0132344821.

[14] D. K. Barry. Service-oriented architecture (SOA) definition. Barry \& Associates, 2000.

http://www.service-architecture.com/articles/ web-services/service-oriented_architecture_soa_ definition.html

[15] SearchSOA.com: Definition service-oriented architecture (SOA). 2003.

http://searchsoa.techtarget.com/definition/ service-oriented-architecture

[16] T. Erl. What is SOA? An Introduction to the Service-Oriented Computing. Goals and Benefits of Service-Oriented Computing. 2016. http://www. whatissoa.com/p16.php
[17] I. Barin. Thermochemical Data of Pure Substances. Weinheim, 1993. ISBN 3-527-28531-8.

[18] M. W. Chase. NIST-JANAF Thermochemical Tables. Journal of Physical and Chemical Reference Data, Monograph 9, 2000. http://kinetics.nist.gov/janaf//

[19] B. J. McBride, M. J. Zehe, S. Gordon. NASA Glenn Coefficients for Calculating Thermodynamic Properties of Individual Species. NASA TPÜ2002-211556, 2002. http://www.archive.org/details/nasa_techdoc_ 20020085330

[20] Department of Chemistry University of Oxford: Chemistry and related Information on the Internet Chemistry link collections. 2008. http:

//www.chem.ox.ac.uk/cheminfo/internet.html

[21] M. J. Zehe. Chemical Equilibrium with Applications. NASA Glenn research center, 2006. http://www.grc. nasa.gov/WWW/CEAWeb/ceaThermoBuild.htm.

[22] C. V. Bale, E. Beliste. Fact-Web suite of interactive programs. 2016. http://www.factsage.com/

[23] GWB: The Geochemist's Workbench. 2016. https: //www.gwb.com/.

[24] HSC-Chemistry-9: Software for Process simulation, Reactions Equations, Heat and Material Balance, Equilibrium Calculation, Electrochemical Cell Equilibriums, Eh-pH Diagrams - Pourbaix diagram. 2016. http://www.hsc-chemistry.net/.

[25] M. S. Ghiorso, R. O. Sack. MELTS Software for thermodynamic modeling of phase equilibria in magmatic systems. 2015. http://melts.ofm-research.org/

[26] R. H. Davies, A. T. Dinsdale, J. A. Gisby, et al. MTDATA - Thermodynamic and Phase Equilibrium Software from the National Physical Laboratory. 2007. http://www.npl.co.uk/upload/pdf/mtdata_calphad_ paper.pdf.

[27] METSIM: The Premier Steady-State 83 Dynamic Process Simulator. 2016. http://www.metsim.com/

[28] CTserver: Computational Thermodynamics (CT) Server. 2016. http://ctserver.ofm-research.org/

[29] Chemical-portal: Chemistry Online Education. 2016. http://www.webqc.org/.

[30] S. Bhattacharjee. TEST - The Expert System for Thermodynamics. Web Portal for Thermodynamic Property Evaluation and Thermal Systems Analysis, 2016. http://test.sdsu.edu/testhome/index.html.

[31] R. C. Berman. Internally-consistent thermodynamic data for minerals in the system Na2O-K2O-CaO-MgOFeO-Fe2O3-Al2O3-SiO2-TiO2-H2O-CO2. Journal of Petrology 29(2):445-522, 1988. DOI:10.1093/petrology/29.2.445

[32] P. Papale. Modeling of the solubility of a two-component $\mathrm{H} 2 \mathrm{O}+\mathrm{CO} 2$ fluid in silicate liquids. American Mineralogist 84:477-492, 1999. DOI:10.2138/am-1999-0402

[33] M. S. Ghiorso, B. W. Evans. Thermodynamics of rhombohedral oxide solid solutions and a revision of the Fe-Ti two-oxide geothermometer and oxygen-barometer. American Journal of Science 308:957-1039, 2008. DOI:10.2475/09.2008.01. 
[34] Solid Earth and Environment GRID: SEEGrid>AnalyticalGeoscience Web $>$ Thermodynamics. 2010. https://www.seegrid.csiro.au/wiki/ AnalyticalGeoscience/Thermodynamics

[35] X. Dong, K. E. Gilbert, R. Guha, et al. Web service infrastructure for chemoinformatics. Journal of Chemical Information and Modeling 47(4):1303-1307, 2007. DOI:10.1021/ci6004349.

[36] B. J. McBride, S. Gordon, M. A. Reno. Coefficients for Calculating Thermodynamic and Transport Properties of Individual Species. NASA TMÜ4513, 1993. http://ntrs.nasa.gov/archive/nasa/casi.ntrs . nasa.gov/19940013151.pdf

[37] C. P. Paolini, S. Bhattacharjee. A web service infrastructure for thermochemical data. Journal of Chemical Information and Modeling 48(7):1511-1523, 2008. DOI:10.1021/ci700457p

[38] S. Bhattacharjee, C. P. Paolini, M. Patterson. A web service infrastructure and its application for distributed chemical equilibrium computation. Journal of Computational Science Education 3(1):19-27, 2012. DOI:10.22369/issn.2153-4136/3/1/3

[39] K. I. Schuchardt, B. I. Didier, T. Elsethagen, et al. Basis set exchange: A community database for computational sciences. Journal of Chemical Information and Modeling 47(4):1045-1052, 2007. DOI:10.1021/ci600510j

[40] C. Steinbeck, S. Kuhn, M. Floris, E. Willighagen. Recent Developments of the Chemistry Development Kit (CDK) - An Open-Source Java Library for Chemo- and Bioinformatics. 2005. https://www.researchgate. net/profile/Christoph_Steinbeck/publication/ 6987061_Recent_Developments_of_the_Chemistry_ Development_Kit_\%28CDK\%29_-_An_Open-Source_ Java_Library_for_Chemo-_and_Bioinformatics/ links/551d48d20cf2000f8f938c85.pdf

[41] R. Guha, M. T. Howard, G. R. Hutchison, et al. The Blue Obelisk - Interoperability in Chemical Informatics. Journal of Chemical Information and Modeling 46(3):991-998, 2006. DOI:10.1021/ci050400b

[42] C. Manuali, A. Lagana. Grif: A new collaborative framework for a web service approach to grid empowered calculations. Future Generation Computer Systems 27(3):315-318, 2010. DOI:10.1016/j.future.2010.08.006
[43] E. Garcia, C. Sanchez, A. Saracibar, et al. A detailed comparison of centrifugal sudden and $\mathrm{j}$ shift estimates of the reactive properties of the $\mathrm{N}+\mathrm{N} 2$ reaction. Physical Chemistry Chemical Physics 48(11):11456-11462, 2009. DOI:10.1039/B915409D

[44] P. Horovčák, D. Dugáček, P. Cirbes. Interpretácia chemických vzorcov pomocou webovej služby [Interpretation of chemical formulae using web service]. Chemické listy 104(11):1029-1033, 2010. http://chemicke-listy.cz/common/content-issue _ 11-volume_104-year_2010.html

[45] E. A. Brizuela. Computing Mixture Temperature from Enthalpy Using NASA Polynomials. 2000. http://www.industrial.combustion.ifrf.net/ paper_download.html?paperId=19

[46] A. Burcat, B. Ruscic. Third Millennium Ideal Gas and Condensed Phase Thermochemical Database for Combustion with Updates from Active Thermochemical Tables. Technion-Israel Institute of Technology, TAE 960, 2005. http:

//www.ipd.anl.gov/anlpubs/2005/07/53802.pdf

[47] American Chemical Society: CAS REGISTRYSM and CAS Registry Numbers. 2016. http://www.cas. org/expertise/cascontent/registry/regsys.html

[48] NIST WebBook Chemie: NIST Standard Reference Database Number 69. 2016. http://webbook.nist.gov/chemistry//

[49] Chemical Book. 2016. http://www.chemicalbook. com/Search_EN . aspx?keyword=1066-33-7

[50] Molbase: Chemical compounds data including chemical structures, properties, synthetic routes, MSDS and NMR spectra. Shanghai MOLBASE Technology Co.,Ltd, 2016. http://www.molbase.com/

[51] EuroChem: Professional Chemistry Guide. 2016. http://www.eurochem.cz/index.php

[52] Environmental, ALS: Analytical Services. 2016. http://www.caslab.com/

[53] Sigma-Aldrich: A Part of Merck. 2016. http: //www.sigmaaldrich.com/united-kingdom.html

[54] Landolt Börnstein Substance / Property Index. 2009. http:

//lb.chemie.uni-hamburg.de/search/index.php

[55] JpGraph: Most powerful PHP-driven charts. Asial Corporation, 2016. http://jpgraph.net/. 